\title{
Dielectric and Ferroelectric Properties of PZN-4.5PT Nanoparticles Thin Films on Nanostructured Silicon Substrate for Ferrophotovoltaic and Energy Storage Application
}

\author{
Rémi Ndioukane1, Moussa Touré1, Diouma Kobor¹, Laurence Motte², Jeanne Solard3, \\ Laurent Lebrun ${ }^{4}$

\begin{abstract}
${ }^{1}$ Laboratoire de Chimie et de Physique des Matériaux (LCPM), University Assane Seck of Ziguinchor, Ziguinchor, Sénégal
${ }^{2}$ Laboratory for Vascular Translational Science (LVTS), University Paris 13, Paris, France

${ }^{3}$ Centrale de Proximité en Nanotechnologies de Paris Nord, Paris, France

${ }^{4}$ Laboratoire Génie Electrique et Ferroélectricité, Institut National des Sciences Appliquées de Lyon, Villeurbanne, France

Email: r.ndioukane1532@zig.univ.sn
\end{abstract}

How to cite this paper: Ndioukane, R., Touré, M., Kobor, D., Motte, L., Solard, J. and Lebrun, L. (2019) Dielectric and Ferroelectric Properties of PZN-4.5PT Nanoparticles Thin Films on Nanostructured Silicon Substrate for Ferrophotovoltaic and Energy Storage Application. Journal of Modern Physics, 10, 613-623.

https://doi.org/10.4236/jmp.2019.106043

Received: November 10, 2018

Accepted: May 13, 2019

Published: May 16, 2019

Copyright $\odot 2019$ by author(s) and Scientific Research Publishing Inc. This work is licensed under the Creative Commons Attribution International License (CC BY 4.0).

http://creativecommons.org/licenses/by/4.0/

\section{(c) (i) Open Access}

\begin{abstract}
The integration of ferroelectric materials as thin films has attracted considerable attention these last years thanks to their outstanding performances that allow considering new features for the realization of photovoltaic devices. Our study focuses on investigating structural, dielectric and ferroelectric properties of undoped and Mn doped PZN-4.5PT nanoparticles thin films on Silicon substrate. We fabricate very stable PZN-4.5PT nanoparticles thin films deposited on nanostructured silicon substrate with giant relative dielectric permittivity of $2.76 \times 10^{4}$ and $17.7 \times 10^{4}$ for respectively the undoped and Mn doped thin films. These values are very large compared to those found in single crystals and might be explained by the influence of the gel in which nanoparticles were dispersed. The SEM images show the crystallization of new hexagonal phases on the film surface probably coming from interaction between $\mathrm{Si}$ and the gel. The hysteresis loops permitted to determine the spontaneous polarization $\left(\mathrm{P}_{\mathrm{s}}\right)$, remnant polarization $\left(\mathrm{P}_{\mathrm{r}}\right)$ and coercive field $\mathrm{E}_{\mathrm{c}}$ which are equal to $11.73 \mu \mathrm{C} / \mathrm{cm}^{2}, 10.20 \mu \mathrm{C} / \mathrm{cm}^{2}$ and 20 $\mathrm{V} / \mathrm{cm}$, respectively for the undoped nanoparticles thin film and 22.22 $\mu \mathrm{C} / \mathrm{cm}^{2}, 19.32 \mu \mathrm{C} / \mathrm{cm}^{2}$ and $20 \mathrm{~V} / \mathrm{cm}$ respectively for the $\mathrm{Mn}$ doped one. These values are high and correspond to the best ones found in literature compared to typical ferroelectric thin films.
\end{abstract}




\section{Keywords}

PZN-PT, Thin Film, Ferroelectric, Perovskite, Nanoparticles, Gel

\section{Introduction}

For semiconductor photovoltaic materials, photons with energy higher than the band gap are absorbed to produce electron-hole pairs which are separated by the internalfield in the p-n junction and collected by the electrodes. Consequently, the photo-induced voltage is limited by the energy barrier height at the interface region and usually smaller than the semiconductor band-gap [1]. Therefore, the ferroelectric photovoltaic effect has been attracted a great deal of attention due to its efficient polarization-governed mechanism of charge separation and ability to generate (open circuit voltage) above band-gap voltages [2].

Recently, the discovery of perovskite ferroelectric nanomaterials opened up a great possibility of application for ferroelectric devices. There have been various nanostructures such as nanoparticles, nanorods, and nano-patterned structures using $\mathrm{PZT}, \mathrm{BaTiO}_{3}$, and $\mathrm{SrTiO}_{3}$ [3] [4] [5]. Those ferroelectric nanomaterials exhibit very different characteristics in its domain dynamics, coercive field and spontaneous polarization response [6] [7] [8] [9]. Ferroelectric lead compounds having a perovskite structure, such as $\mathrm{Pb}\left(\mathrm{Zn}_{1 / 3} \mathrm{Nb}_{2 / 3}\right) \mathrm{O}_{3}(\mathrm{PZN})$ [10], $\mathrm{Pb}\left(\mathrm{Mg}_{1 / 3} \mathrm{Nb}_{2 / 3}\right) \mathrm{O}_{3}$ (PMN) [11], and their solid solutions with $\mathrm{PbTiO}_{3}(\mathrm{PT})$, have been investigated for high performance ultrasonic transducer applications [12] [13] [14] [15] [16]. These solid solutions have excellent piezoelectric and ferroelectric properties in single crystal form compared to the above ferroelectrics. In addition, its high relative dielectric permittivity value could permit to gain a very high open circuit voltage in photovoltaic cells thanks to the charge accumulation process. Then PZN-PT could be one of the promising new materials for such ferrophotovoltaic devices. Indeed, these last years, high levels of papers have been published about PMN-PT and PZN-PT single crystals [17] [18] [19] [20], showing at least ferroelectric, ferroelastic and piezoelectric properties 10 times higher than those of PZT. However, there is no publication on PZN-PT as thin films for ferrophotovoltaic effect. We know that the greatest difficulty to use such single crystals on electronic devices is to achieve them in thin layers form because of their incongruent melting property. If we fabricate the nanoparticles thin film of such materials without losing ferroelectricity or photovoltaic properties, we can expand the ferroelectric device to various substrates, structures, and nano-scale applications.

In this paper, to integrate them into silicon nanostructures, we realized PZN-4.5PT nanoparticles deposition, already synthesized by the so-called solution flux method [19], as thin film on p-type $<100>$ oriented nanoporous silicon substrate. Surface morphology, dielectric and ferroelectric properties of as deposited thin films were investigated. 


\section{Experimental Procedure}

\subsection{PZN-PT Nanoparticles Thin Film Fabrication and Surface Characterization}

Undoped and 1\% Mn doped PZN-4.5PT grounded powders were dispersed in gel fabricated in the laboratory LCPM in Assane Seck University of Ziguinchor. To obtain a homogeneous film, spinning process was carried out at room temperature using a spin coater Midas $1200 \mathrm{D}$ at $3500 \mathrm{rpm}$ with an initial acceleration time of 5 seconds and an operating time of $10 \mathrm{~min}$. After $10 \mathrm{~min}$ bake in oven at $100^{\circ} \mathrm{C}$, thermal annealing in a $\mathrm{K} 114$ type muffle furnace was performed. The heating rate of the furnace is chosen between 10 and $20^{\circ} \mathrm{C} / \mathrm{min}$. A $30-60$ min plateau was carried out at $900^{\circ} \mathrm{C}$ for the gel diffusion through the p-type silicon $(<100>$ oriented Boron doped monocrystalline silicon with resistivity $5-10$ $\Omega . c m$ and thickness of $600-650 \mu \mathrm{m}$ ) nanowires substrate. Cooling at ambient temperature was done naturally. Different samples were fabricated using the two nanopowders and were characterized. Surface morphology of thin films samples were observed using an electron beam lithography system Pioneer Raith model in $\mathrm{C}(\mathrm{PN}) 2$ (Paris 13 University).

\subsection{Dielectric and Ferroelectric Characterization}

For dielectric properties characterization, the sample with the deposit thin film was metallized on the both sides with silver paste using the screen-printing method. In order to have good adhesion between the silver and the thin layer, the metallized sample was annealed at $450^{\circ} \mathrm{C}$ for $30 \mathrm{~min}$ in a thermo scientific model FB1310M-33 oven. For dielectric losses measurements and to calculate the relative dielectric constant $\left(\varepsilon_{r}\right)$, the capacity measurements as function of the temperature and the frequency were performed using a LCR meter model LCR-819 from GWINSTEK. The relative dielectric constant was determined using the following formula

$$
\varepsilon_{r}=\frac{C e}{S \varepsilon_{0}}
$$

where $C$ is the measured capacity, e the thickness, $S$ the metallized surface and $\varepsilon_{0}$ the vacuum permittivity $\left(8.85 .10^{-12} \mathrm{~F} / \mathrm{m}\right)$. A modified Sawyer-Tower circuit at room temperature was used to measure the polarization versus the DC field. From polarization- $\mathrm{E}_{\text {field }}$ curves, $\mathrm{E}_{\mathrm{c}}, \mathrm{P}_{\mathrm{r}}$ and $\mathrm{P}_{\mathrm{s}}$ were determined.

\section{Results and Discussion}

\subsection{Surface Characterization}

Figure 1 shows the SEM images of the different thin layers revealing that the nanoparticles gel is diffused inside the nanowires and covered totally the whole surface. The images of Figure 1(a)-(c) (increasing magnetization) show the complex nature of the undoped nanoparticles thin films randomly distributed. One can find the presence of black zones $\left(\mathrm{Pb}\right.$ is highly reactive with $\mathrm{Si}$ at $600^{\circ} \mathrm{C}$ 


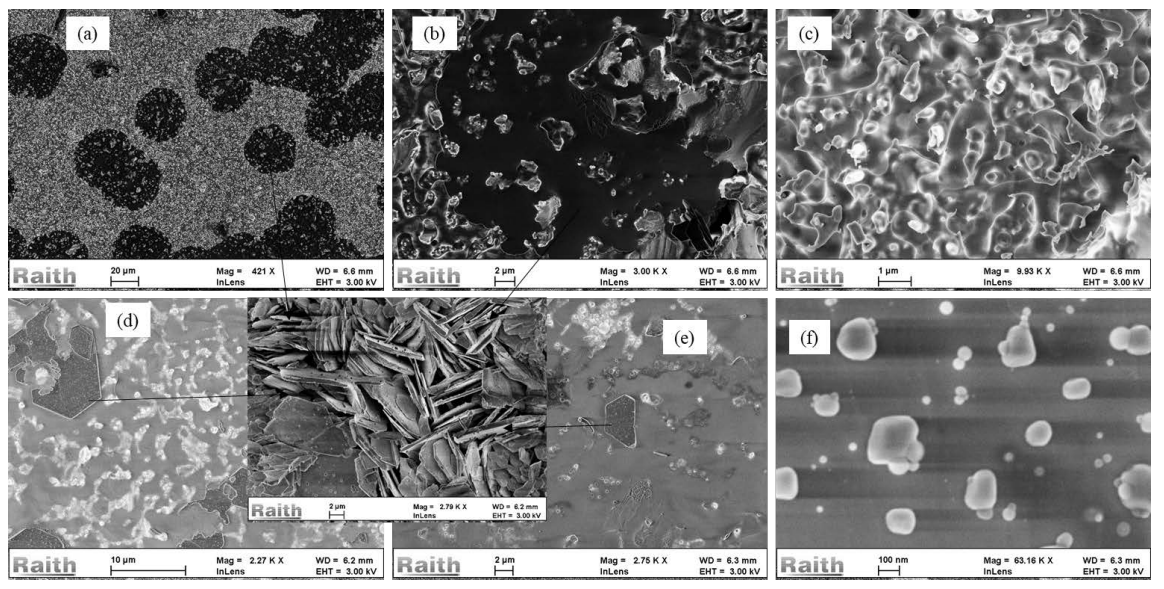

Figure 1. SEM images of (a) normal, (b) zooming on black circle and (c) zooming on gray zone for undoped; (d) normal zone 1, (e) normal zone 2 and (f) zooming on zone for $1 \% \mathrm{Mn}$ doped PZN-4.5PT nanoparticles thin layers on $<100>$ oriented silicon substrate.

to give a hexagonal nano-crystals; on the other hand it is possible that there is a reaction between $\mathrm{Pb}$ and the gel giving these nano-crystals), which could be explained by the formation of a new phase and due to the presence of new atoms from the gel containing nanoparticles such as $\mathrm{Pb}$ and $\mathrm{P}$ that may form new hexagonal complexes with Si thanks to annealing. In Figure 1(c) top terminals of silicon nanowires could be seen. Figure 1(d) to Figure 1(f) show homogeneous distribution of $1 \% \mathrm{Mn}$ doped PZN-PT nanoparticles. However, it is interesting to notice that the local black circles are composed by hexagonal shapes nanocrystals (Figure 1(d), Figure 1(e) and the zooming image). Figure 1(f) shows the distribution of the doped nanoparticles on the surface with sizes varying from less than $10 \mathrm{~nm}$ to around $200 \mathrm{~nm}$.

\subsection{Dielectric and Ferroelectric Characterization}

Figure 2 shows the relative dielectric permittivity and dielectric losses as function of temperature. These curves reveal the presence of two transition temperatures (ferro-ferro and ferro-para). The first transition $\left(\mathrm{T}_{\mathrm{RT}}=130^{\circ} \mathrm{C}\right)$ is assigned to a ferroelectric to ferroelectric phase transition which could be from a Rhombohedral and hexagonal mixte ferroelectric phase (perovskite nanoparticles and hexagonal nanocrystals) to another ferroelectric phase such as tetragonal or total hexagonal one. Doping did not affect this transition value and the value is closer to the bulk PZN-4.5PT crystals $\left(130^{\circ} \mathrm{C}[19]\right)$. The second peak (around $310^{\circ} \mathrm{C}$ ) corresponds to the transition from ferroelectric to paraelectric $\left(\mathrm{T}_{\mathrm{c}}\right)$. According to Figure 2(a) and Figure 2(b), showing permittivity and dielectric losses temperature dependence, for undoped and $\mathrm{Mn}$ doped PZN-4.5PT nanoparticles thin layers, Tc increased from $180^{\circ} \mathrm{C}$ [19] to $310^{\circ} \mathrm{C}$ respectively for bulk and for nanoparticles thin layers due to, probably, the gel effect. Relative dielectric permittivity (Table 1 ) values are very high at ambient temperature and $1 \mathrm{kHz}\left(10^{4}\right.$ to $10^{6}$ for undoped and $10^{4}$ to $10^{5}$ for doped nanoparticles) compared to the bulk one with $10^{3}$ to $6 \times 10^{3}$. This remarkable increase in the dielectric permittivity 
Table 1. Dielectric and transitions parameters of the undoped and Mn doped nanoparticles thin layers.

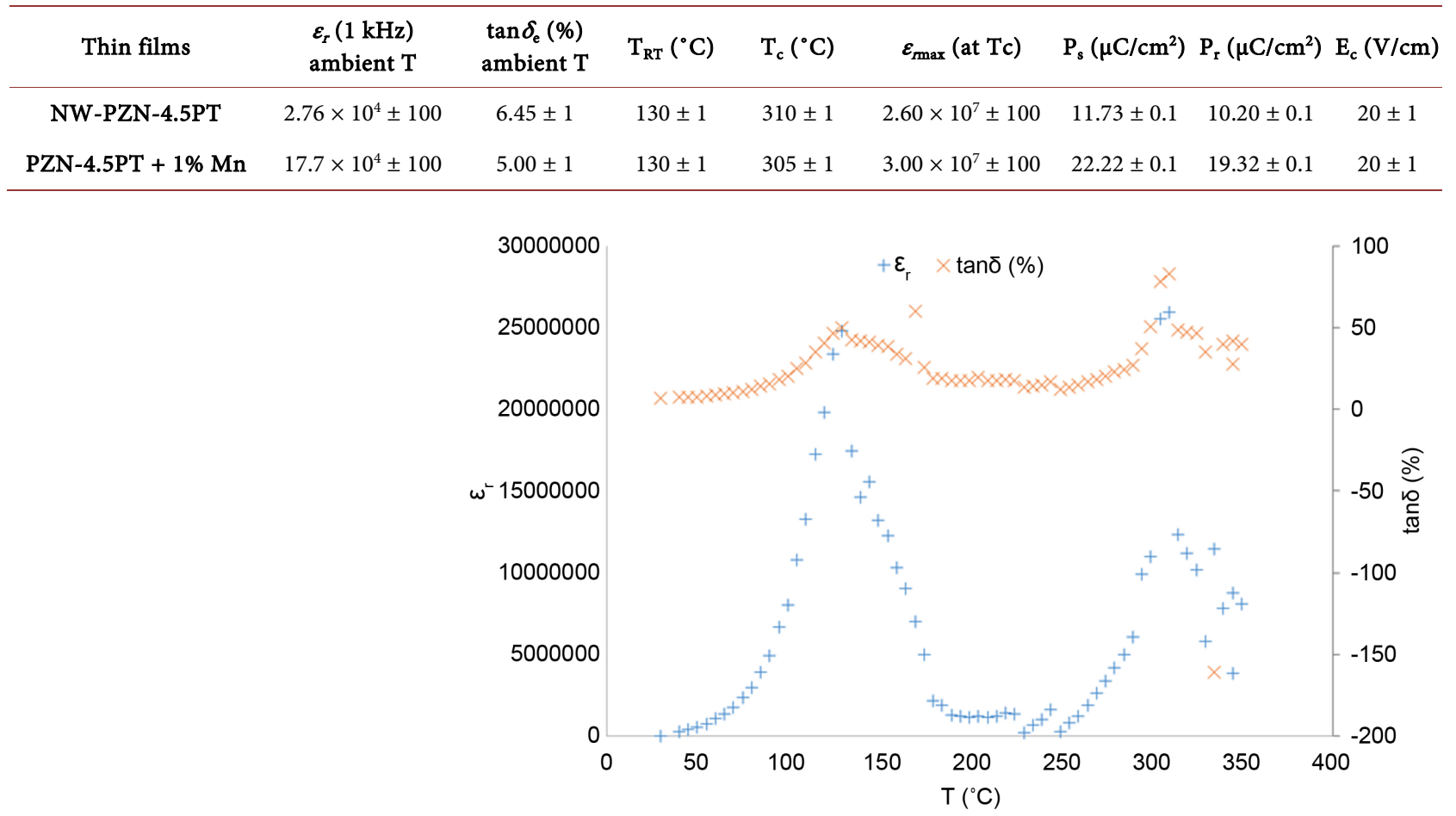

(a)

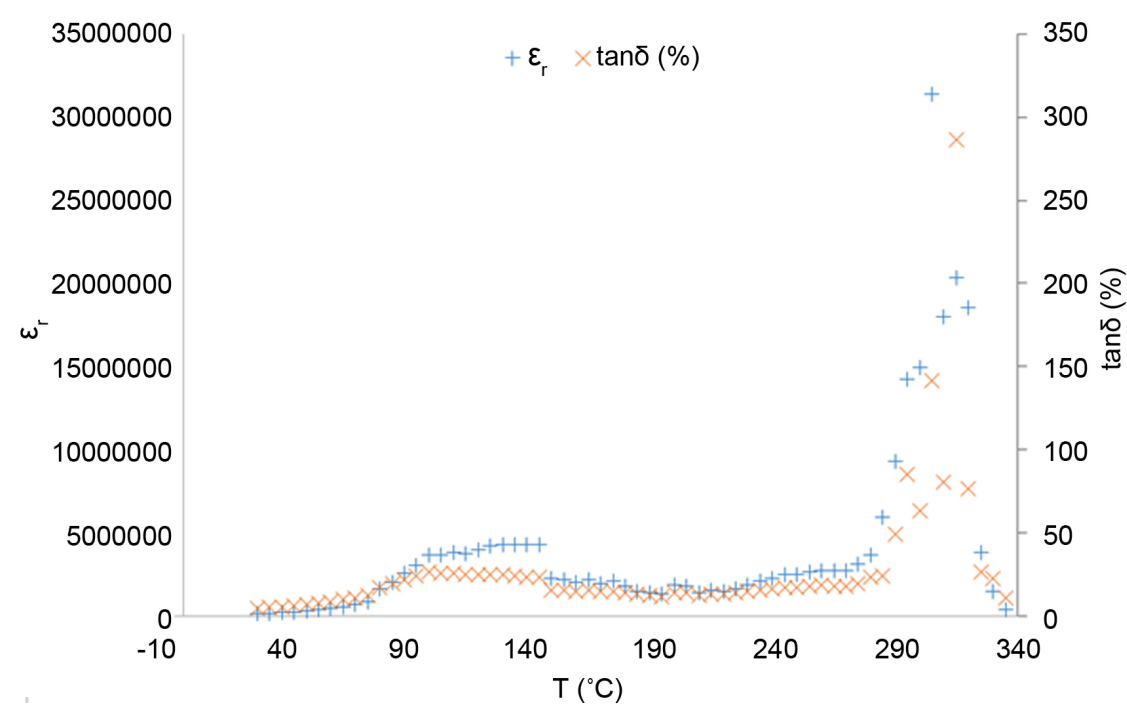

(b)

Figure 2. Relative dielectric permittivity and dielectric losses versus temperature for (a) undoped and (b) 1\% Mn doped PZN-4.5PT nanoparticles thin films deposited on $<100>$ oriented silicon nanowires substrate.

could be explained by the new phase from combination of Si-perovskite-gel overgrowth on the silicon surface. The dielectric losses are low compared to other high $\mathrm{K}$ materials; their values are equal to $6.45 \%$ and $5 \%$ respectively for the undoped and Mn doped PZN-4.5PT thin films. These values show that the mechanical factor would be very interesting and high. The results from dielectric 
measurements show the potential application of such materials in micro and nanoelectronic devices as a colossal relative dielectric permittivity material.

Figure 3 represents the dielectric permittivity and dielectric losses dependence with applied electric field for undoped and Mn doped PZN-4.5PT thin films. From these curves it is clear that the dielectric permittivity value is very colossal in applied electric field conditions. It increases linearly between $15\left(\varepsilon_{r} \approx\right.$ $\left.1 \times 10^{9}\right)$ to $25 \mathrm{~V} / \mathrm{cm}$ reaching to its maximum value $\left(5 \times 10^{9}\right)$ at $\mathrm{E} \approx 50 \mathrm{~V} / \mathrm{cm}$ for undoped film and from $2 \times 10^{9}$ to $9 \times 10^{9}$ for the doped one (Figure 3(a)). Over $60 \mathrm{~V} / \mathrm{cm}$, the dielectric permittivity dropped down to its lowest values $\left(\varepsilon_{r} \approx 2 \times\right.$ $10^{9}$ and $7 \times 10^{7}$ respectively for the undoped and Mn doped nanoparticles films). Physically, we supposed that increasing the voltage would permit some defaults and impurities to give enough energy for conduction band. So the increase of the material conductivity would certainly decrease permittivity property.

These results show that this new material could support very large electric field change $(40-60 \mathrm{~V} / \mathrm{cm})$ compared to the other thin films dielectric. However,

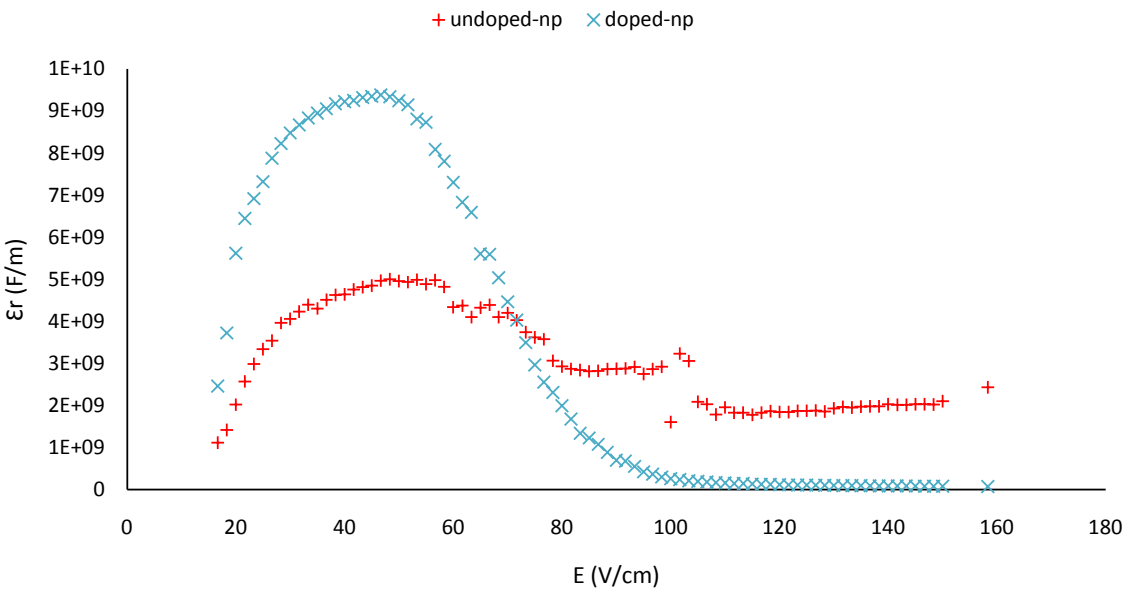

(a)

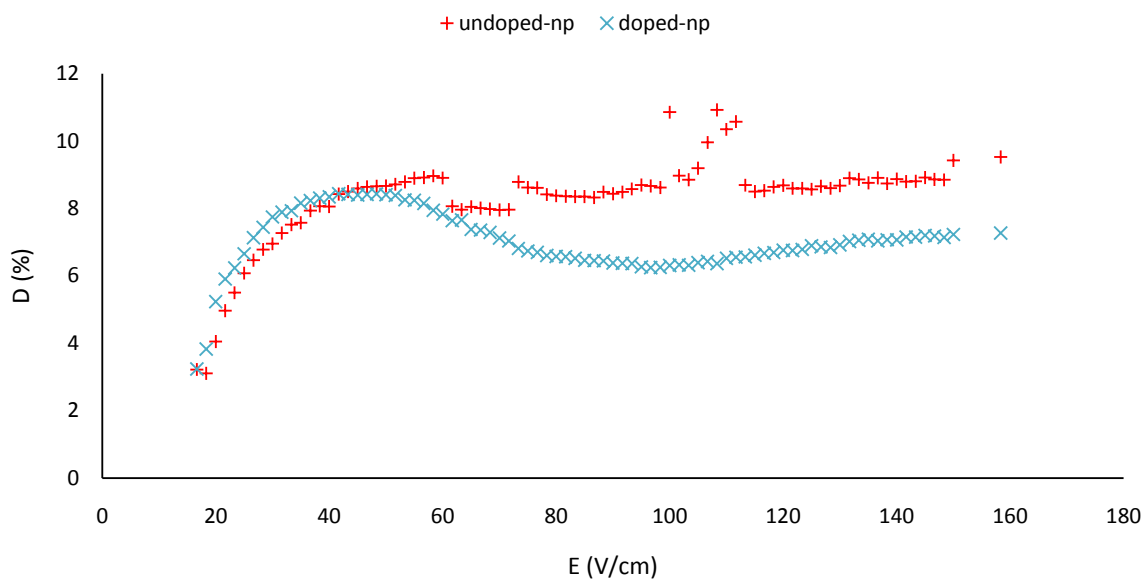

(b)

Figure 3. (a) Relative dielectric permittivity and (b) losses for undoped and $1 \% \mathrm{Mn}$ doped PZN-4.5PT nanoparticles thin films deposited on $<100>$ oriented silicon nanowires versus applied electric field. 
over a certain value its performances would decrease. It exists a critical electric field value (around $60 \mathrm{~V} / \mathrm{cm}$ ) corresponding to the maximum dielectric permittivity for these materials polarization. The Mn doping did not greatly affect the thin films properties. However, it is clear that it stabilizes the electric field dependence (values are more stable compared to undoped). Indeed, one can see in Figure 3(b) that the losses are very stable for applied electric field over $60 \mathrm{~V} / \mathrm{cm}$ with a value equal around $7 \%$ for the doped nanoparticles while it increases suddenly for the undoped one before being stable around $9 \%$.

Figure 4 shows the dielectric permittivity and losses as function of frequency with bias on "off" (Figure 4(a) and Figure 4(c)) and with bias on "on" (Figure $4(\mathrm{c})$ and Figure $4(\mathrm{~d})$ ). We note that the bias is remaining at $0 \mathrm{~V}$. The dielectric measurements are carried out as a function of frequency in the range of $100 \mathrm{~Hz}$ to $100 \mathrm{kHz}$ to explore relaxation effects. The large dielectric constant for undoped thin film decreases continuously with an increase in frequency, which is a typical characteristic of any ferroelectric material. Whereas, for the Mn doped sample, the dielectric constant decreases slowly for the further increase in the frequency.

This kind of behavior usually comes from the space charges at low frequencies. Along with the improvement in the dielectric constant, an increase in the dielectric loss (Figure 4(c)) is also found for the doped nanoparticles thin film. The dielectric permittivity and losses behaviors in Figure 4(a) and Figure 4(b) (under $0 \mathrm{~V}$ bias) are totally different to those from Figure 4(a) and Figure 4(c). Indeed, in the latter we notice the presence of negative values (meaning negative capacity) over a certain apparent resonance frequency (around $10 \mathrm{kHz}$ ) for both materials. These negative values could come from charge injection and also charge accumulation due to the colossal dielectric permittivity. The relative dielectric permittivity values at high frequency are equal to $1.17 \times 10^{4}$ and $3.9 \times 10^{4}$ for respectively undoped and $\mathrm{Mn}$ doped thin films and with bias "off". These values are in the same range that those determined from dielectric permittivity-temperature curves at $1 \mathrm{kHz}$ (Table 1 ). The permittivity values with bias on "on" at $0 \mathrm{~V}$ are negative and equal to $-1.09 \times 10^{6}$ and $-2.23 \times 10^{6}$ respectively for
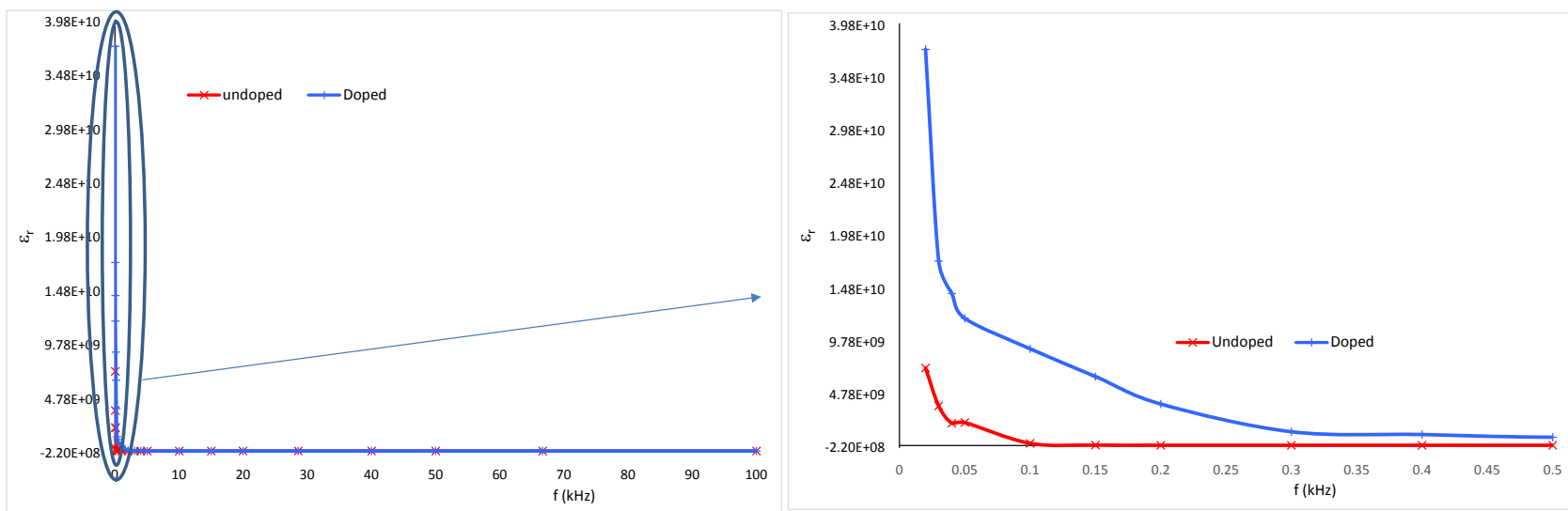

(a) 


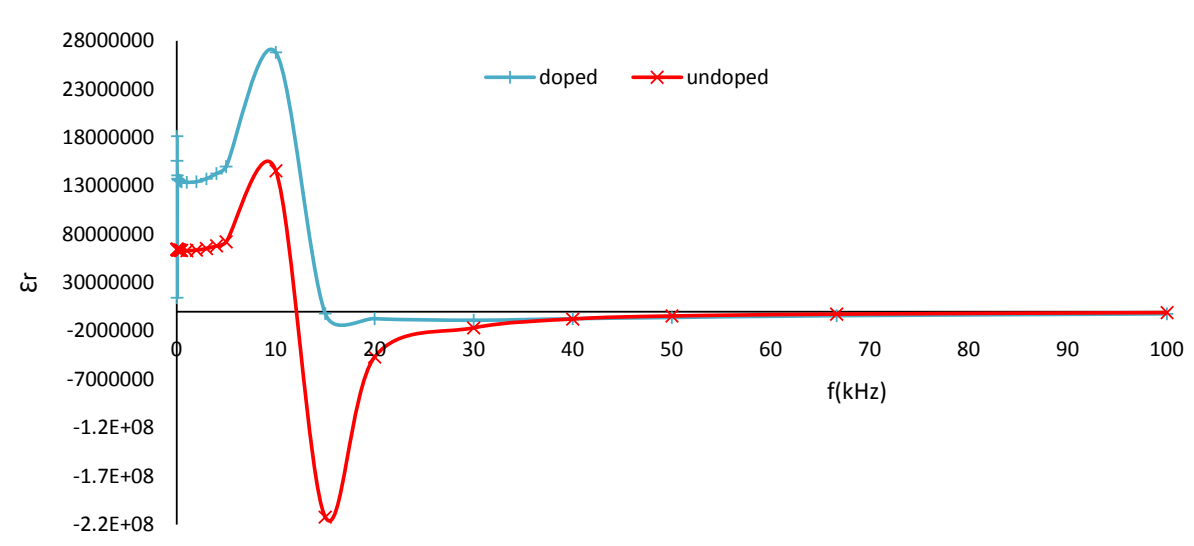

(b)
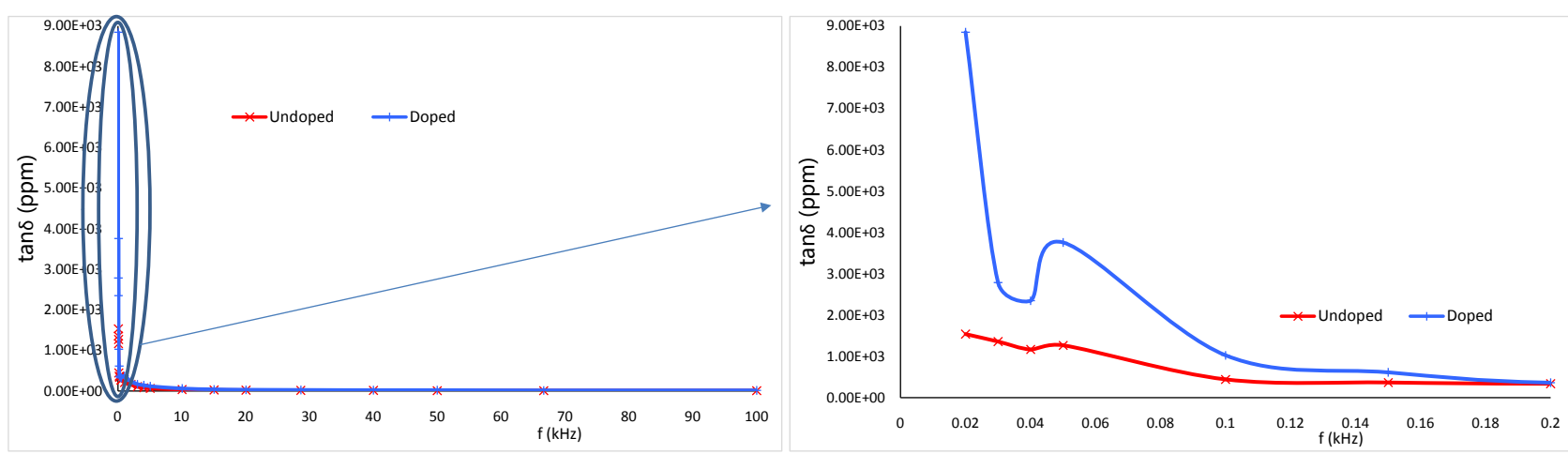

(c)

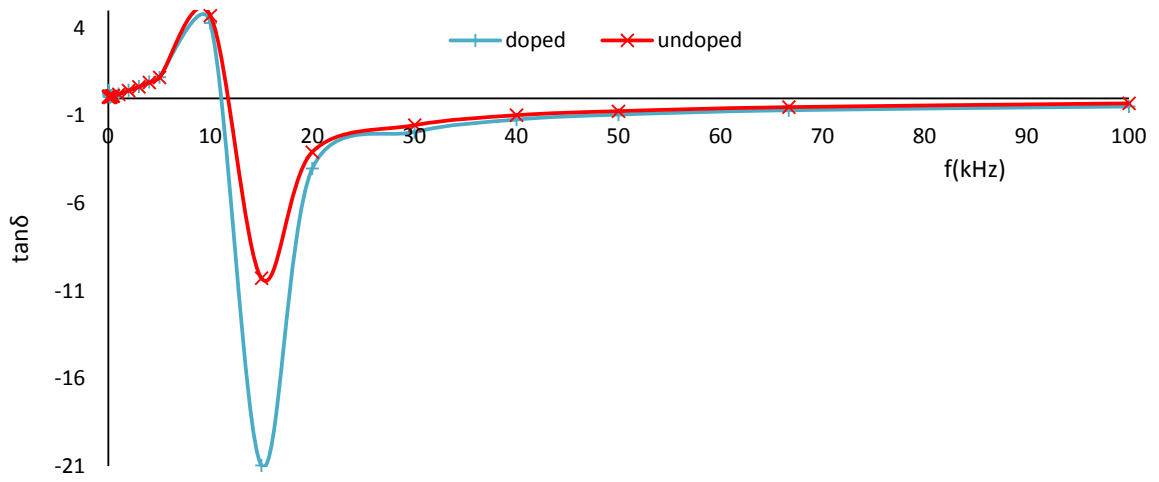

(d)

Figure 4. Dielectric permittivity versus frequency with (a) 0 V bias "on" and (b) bias "off"; dielectric losses (c) 0 V bias "on" and (d) bias "off" for undoped and Mn doped PZN-4.5PT nanoparticles thin films deposited on $<100>$ oriented silicon nanowires.

undoped and Mn doped samples. The presence of bias since it is equal to $0 \mathrm{~V}$ increases highly the permittivity values and behavior both for the two films. As explained above, this could be due to charge injection and accumulation. These large relative dielectric values and low dielectric losses make these materials interesting candidates for ferrophotovoltaic and energy storage.

Figure 5 shows the room temperature dielectric constant and dielectric losses versus applied electric field of the Mn doped PZN-PT nanoparticles thin film. The curve is butterfly shaped, providing the evidence of weak ferroelectricity of 


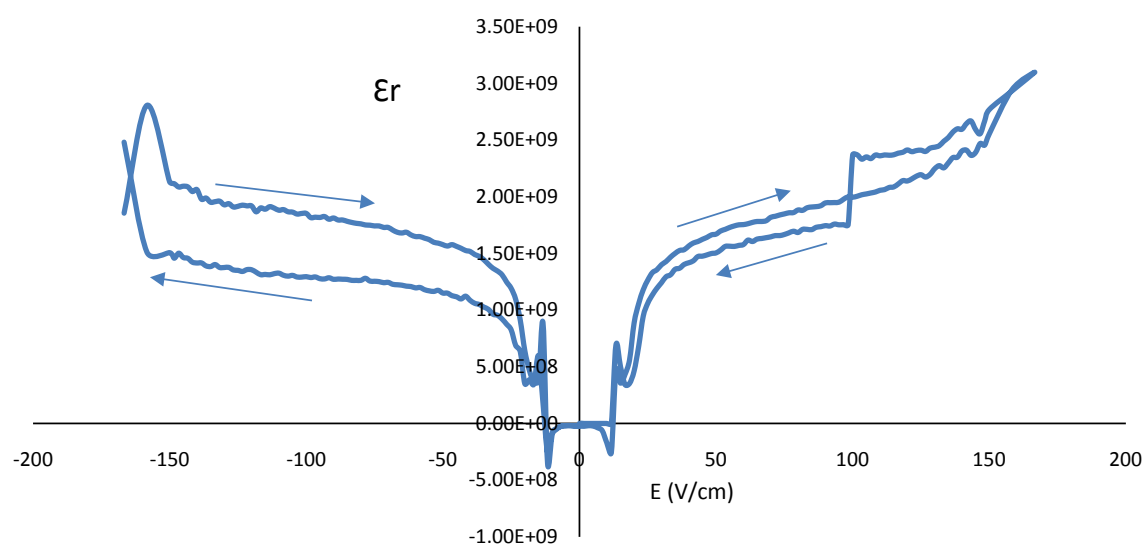

(a)

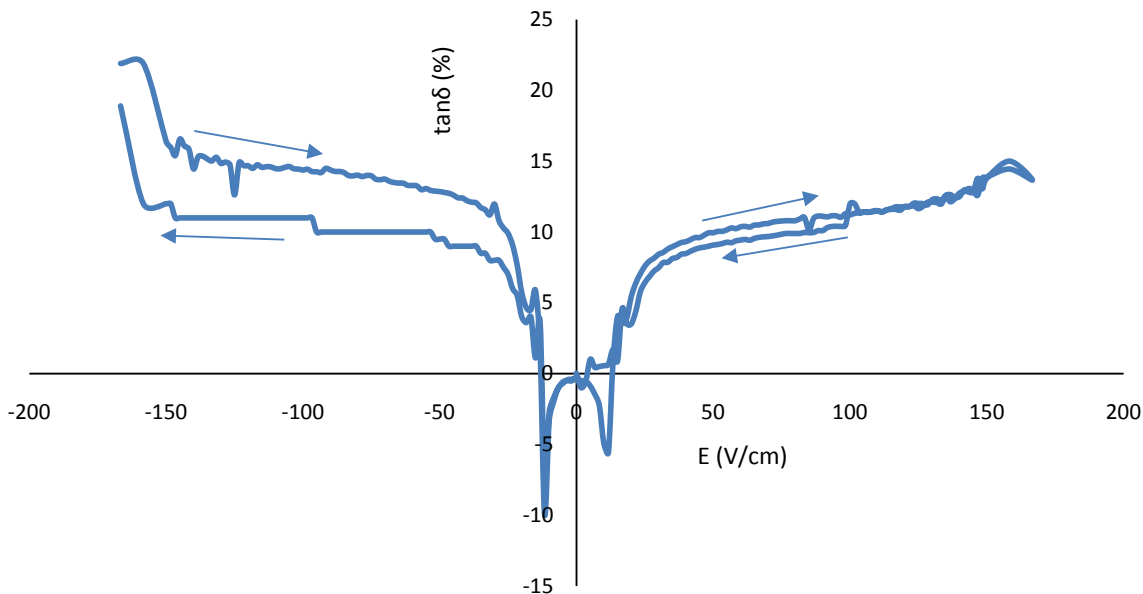

(b)

Figure 5. Completed cycles for (a) relative dielectric permittivity and (b) dielectric losses for $1 \%$ Mn doped PZN-4.5PT nanoparticles thin layers deposited on $<100>$ oriented silicon nanowires substrate.

the PZN-PT thin films at room temperature. The tunability $\left(\left(\varepsilon_{\max }-\varepsilon_{\min }\right) / \varepsilon_{\max }\right)$ of the doped PZN-PT thin films is around $86 \%$. This film showed very high tunability compared to those found in other ferroelectric materials such as the BNT-BT-ST thin films (29\% and 35\% [21]). This enhancement of dielectric constant was attributed to the relatively large hexagonal formed nanocrystals of the thin films due to the reaction between the gel and silicon substrate and also charge accumulation at the thin film and the electrode interface.

Figure 6(a) and Figure 6(c) show the stimulus and the response signals as a function of time. The stimulus triangle wave $(\mathrm{X})$ and the voltage (sine wave) across the sense capacitor $(\mathrm{Y})$ prompt the ferroelectric behavior of the thin films. The film capacitor is modulating the stimulus wave. The modulation by the ferroelectric capacitor is particularly apparent in Figure 6(b) and Figure 6(d). This figure presents a ferroelectric hysteresis loop by using X:Y mode as explained in the experimental method described in [22]. The values of remnant polarization $\mathrm{P}_{\mathrm{r}}$, spontaneous polarization $\mathrm{P}_{s}$ and coercive field $\mathrm{E}_{\mathrm{c}}$ are equal to $11.73 \mu \mathrm{C} / \mathrm{cm}^{2}$, $10.20 \mu \mathrm{C} / \mathrm{cm}^{2}$ and $20 \mathrm{~V} / \mathrm{cm}$, respectively for the undoped nanoparticles thin film 

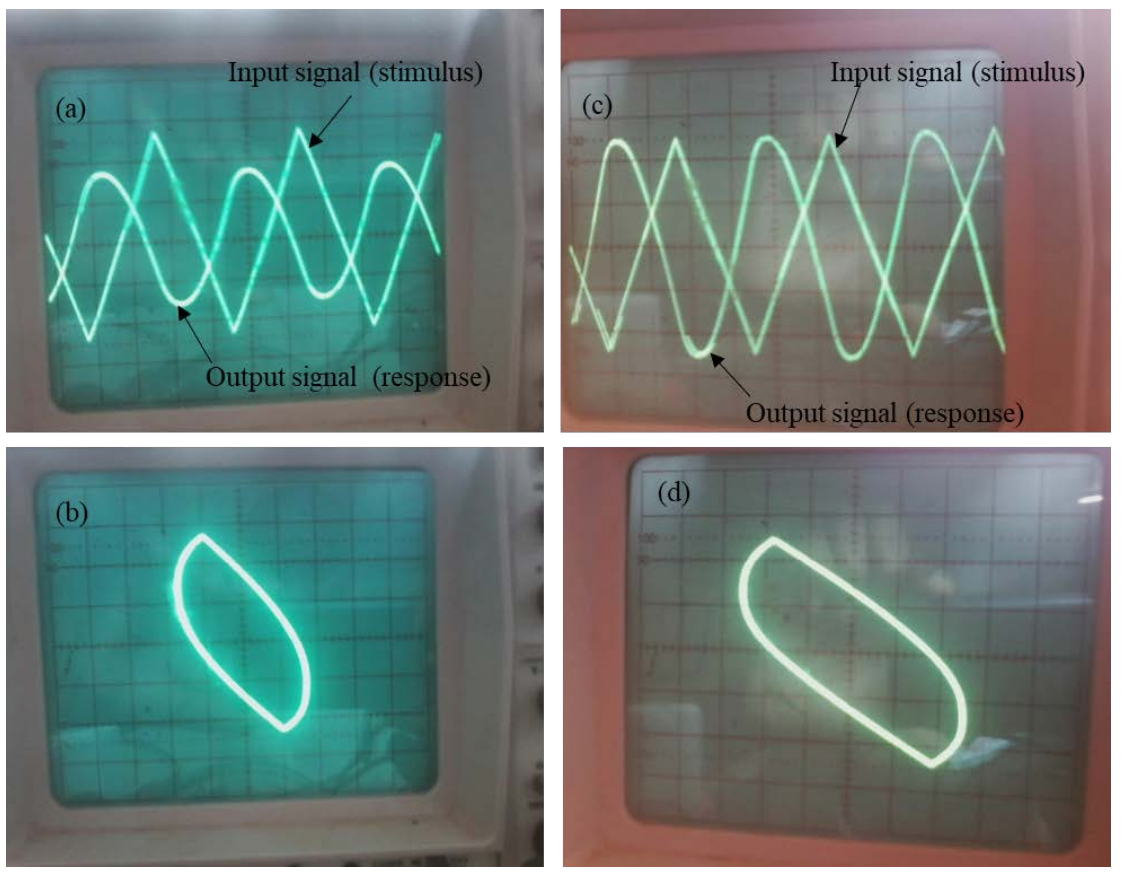

Figure 6. Modulation of the stimulus triangle wave and response (a) and (c) and their ferroelectric hysteresis loops (b) and (d) for respectively undoped and 1\% Mn doped PZN-4.5PT nanoparticles thin layers on $<100>$ oriented silicon nanowires.

and $22.22 \mu \mathrm{C} / \mathrm{cm}^{2}, 19.32 \mu \mathrm{C} / \mathrm{cm}^{2}$ and $20 \mathrm{~V} / \mathrm{cm}$ respectively for the Mn doped thin film. These values are large (high) and correspond to the best ones found in literature compared to typical ferroelectric thin films. One can see that Mn doping increases the polarization values. This is normal compared to the dielectric constant value in room temperature at $1 \mathrm{kHz}$ increasing by doping (Table 1) facilitating charge accumulation and so polarization.

\section{Conclusions}

In this study, we fabricate with success PZN-4.5PT nanoparticles thin films. Their ferroelectric behavior has been demonstrated, measured and evaluated. We found colossal dielectric constant, which could be explained by the presence of new crystals components probably coming from the reaction between $\mathrm{Si}$ and the gel where nanoparticles were dispersed. These results make such thin films very potential candidates for energy storage and for ferrophotovoltaic application.

The determination of the composition of these hexagonal nanocrystals on the film surface and the origin of such colossal relative permittivity would be investigated.

\section{Acknowledgements}

This work is supported by Agence Universitaire de la Francophonie (AUF).

\section{Conflicts of Interest}

The authors declare no conflicts of interest regarding the publication of this paper. 


\section{References}

[1] Qin, M., Yao, K. and Liang, Y.C. (2008) Applied Physics Letters, 93, Article ID: 122904. https://doi.org/10.1063/1.2990754

[2] Fridkin, V.M. (2001) Crystallography Reports, 46, 654-658. https://doi.org/10.1134/1.1387133

[3] Bernal, A., et al. (2012) Advanced Materials, 24, 1160-1165. https://doi.org/10.1002/adma.201103993

[4] Yuriy, G., Olena, Z. and Anatoliy, G. (2012) Emerging Applications of Ferroelectric Nanoparticles in Materials Technologies, Biology and Medicine. In: Peláiz-Barranco, A., Ed., Advances in Ferroelectrics, IntechOpen, Rijeka Craotia, 475-497.

[5] Joshi, U.A. and Lee, J.S. (2005) Small, 1, 1172-1176. https://doi.org/10.1002/smll.200500055

[6] Lang, X.Y. and Jiang, Q. (2007) Journal of Nanoparticle Research, 9, 595-603. https://doi.org/10.1007/s11051-005-9066-1

[7] Varghese, J., Whatmore, R.W. and Holmes, J.D. (2013) Journal of Materials Chemistry C, 1, 2618-2638. https://doi.org/10.1039/c3tc00597f

[8] Michael, T., Trimper, S. and Wesselinowa, J.M. (2006) Physical Review B, 74, Article ID: 214113. https://doi.org/10.1103/PhysRevB.74.214113

[9] Basun, S.A., et al. (2011) Physical Review B, 84, Article ID: 024105. https://doi.org/10.1103/PhysRevB.84.024105

[10] Bokov, V. A. and Myl'nikova, I.E. (1960) Soviet Physics, Solid State, 2, 2428.

[11] Smolenskii, G.A., Isupov, V.A., Agranovskaya, A.I. and Popov, S.N. (1961) Soviet Physics, Solid State, 2, 2584.

[12] Nomura, S., Takahashi, T. and Yokomizo, Y. (1969) Journal of the Physical Society of Japan, 27, 262. https://doi.org/10.1143/JPSJ.27.262

[13] Kuwata, J., Uchino, K. and Nomura, S. (1981) Ferroelectrics, 37, 579-582. https://doi.org/10.1080/00150198108223490

[14] Kuwata, J., Uchino, K. and Nomura, S. (1982) Japanese Journal of Applied Physics, 21, 1298. https://doi.org/10.1143/JJAP.21.1298

[15] Shrout, T.R., Change, Z.P., Kim, N. and Markgraf, S. (1990) Ferroelectrics Letters Section, 12, 63-69. https://doi.org/10.1080/07315179008201118

[16] Mulvihill, M.L., Park, S.E., Risch, G., Li, Z., Uchino, K. and Shrout, T.R. (1996) Japanese Journal of Applied Physics, 35, 3984. https://doi.org/10.1143/JJAP.35.3984

[17] Lebrun, L., Zhang, S., Randall, C.A., Shrout, T.R. and Guyomar, D. (2002) Ceramic Transactions, 136, 117.

[18] Benayad, A., Kobor, D., Lebrun, L., Guiffard, B. and Guyomar, D. (2004) Journal of Crystal Growth, 270, 137-144. https://doi.org/10.1016/j.jcrysgro.2004.06.017

[19] Kobor, D. (2005) Synthesis and Characterization of PZN-4.5PT Single Crystals by Flux Method. Thesis, INSA, Lyon.

[20] Prya, S. and Uchino, K. (2002) Journal of Applied Physics, 91, 4515. https://doi.org/10.1063/1.1459101

[21] Li, W., et al. (2015) Ceramics International, 41, S356-S360. https://doi.org/10.1016/j.ceramint.2015.03.178

[22] Kobor, D., et al. (2015) Processing and Application of Ceramics, 9, 107-115. https://doi.org/10.2298/PAC1502107K 